\title{
Relativistic Green's function approach to charged-current neutrino-nucleus quasielastic scattering
}

\author{
Andrea Meucci, Carlotta Giusti, and Franco Davide Pacati \\ Dipartimento di Fisica Nucleare e Teorica, Università degli Studi di Pavia and \\ Istituto Nazionale di Fisica Nucleare, Sezione di Pavia, I-27100 Pavia, Italy
}

(Dated: November 1, 2018)

\begin{abstract}
A relativistic Green's function approach to inclusive quasielastic charged-current neutrino-nucleus scattering is developed. The components of the hadron tensor are written in terms of the singleparticle Green's function, which is expanded on the eigenfunctions of the nuclear optical potential, so that final state interactions are accounted for by means of a complex optical potential but without a loss of flux. Results for the $\left(\nu_{\mu}, \mu^{-}\right)$reaction on ${ }^{16} \mathrm{O}$ and ${ }^{12} \mathrm{C}$ target nuclei are presented and discussed. A reasonable agreement of the flux-averaged cross section on ${ }^{12} \mathrm{C}$ with experimental data is achieved.
\end{abstract}

PACS numbers: 25.30.Pt: Neutrino scattering, 13.15.+g: Neutrino interactions, 24.10.Jv: Relativistic models, 24.10.Cn: Many-body theory

\section{INTRODUCTION}

The reactions with an incident neutrino interacting with a complex nucleus have gained in recent years a wide interest, owing both to astrophysical reasons and to the aim of investigating the neutrino properties with a high accuracy. Besides the measurements with large underground detectors, some experiments have also been performed 1, 2, 3] using a pion beam which weakly decays producing leptons. In this case the most part of the neutrinos which are obtained is related to muons, with a smaller component of electron neutrinos.

Both weak neutral- and charged-current scattering have stimulated detailed investigations. In particular, we are here interested in charged-current reactions at an energy below $1 \mathrm{GeV}$ as they have shown to be dependent on nuclear structure effects. Different approaches have been applied to investigate such processes, including the so-called "elementary particle model" [4], random phase approximation (RPA) in the framework of a relativistic Fermi gas model [5] or a Fermi gas model with local density approximation [6], shell model 7, 8] and relativistic shell model 9], RPA among quasiparticles [8] and continuum RPA [10, 11]. The reaction goes through a quasielastic (QE) mechanism, where the neutrino interacts with one single neutron and a proton together with a negative muon are emitted. The effect of final state interactions (FSI) has been stressed to significantly contribute to the cross section [12] and has been calculated with a relativistic shell model including a phenomenological optical potential, which describes the interaction of the outgoing nucleon with the residual nucleus, with and without imaginary part 13.

The optical potential is fitted to reproduce the elastic proton-nucleus scattering through its real component, while its imaginary part takes into account the scattering towards the inelastic channels. This means that the reaction channels are globally described by a loss of flux produced by the imaginary part of the complex poten- tial. This model has been applied with great success to exclusive QE electron scattering [14], where it is able to explain the experimental cross sections of one-nucleon knockout reactions in a range of nuclei from carbon to lead. In an inclusive process, however, where some of the reaction products are not detected and the inelastic channels are also included in the experimental cross section, the flux must be conserved. This fact is sometimes described by dropping the imaginary part of the optical potential. This procedure conserves the flux but it is not consistent with the exclusive reaction, which can only be reproduced with a careful treatment of the optical potential, including both real and imaginary parts [14].

In this paper we apply a Green's function approach where the conservation of flux is preserved and FSI are treated in the inclusive reaction consistently with the exclusive one. This method was discussed in a nonrelativistic 15] and in a relativistic [16] framework for the case of inclusive electron scattering and it is here adapted, in a relativistic framework, to charged-current neutrino scattering. In this approach, the components of the nuclear response are written in terms of the single-particle optical-model Green's function. This result was originally derived by arguments based on the multiple scattering theory [17] and successively by means of the Feshbach projection operator formalism [15, 18, 19, 20]. The spectral representation of the single-particle Green's function, based on a biorthogonal expansion in terms of the eigenfunctions of the non-Hermitian optical potential, allows one to perform explicit calculations and to treat FSI consistently in the inclusive and in the exclusive reactions. Important and peculiar effects are given in the inclusive $\left(e, e^{\prime}\right)$ reaction by the imaginary part of the optical potential, which is responsible for the redistribution of the strength among different channels.

In Sec. II the general formalism of the charged-current neutrino-nucleus scattering is given. In Sec. III, the Green's function approach is briefly reviewed. In Sec. $\mathrm{IV}$, the results obtained on ${ }^{16} \mathrm{O}$ and ${ }^{12} \mathrm{C}$ target nuclei are 
presented and discussed. Some conclusions are drawn in Sec. V.

\section{THE INCLUSIVE CROSS SECTION}

In a charged-current process neutrinos and antineutrinos interact with nuclei via the exchange of weak-vector bosons and charged leptons are produced in the final state. The cross section of an inclusive reaction where an incident neutrino or antineutrino, with four-momentum $k_{i}^{\mu}=\left(\varepsilon_{i}, \boldsymbol{k}_{i}\right)$, is absorbed by a nucleus and only the outgoing lepton, with four-momentum $k^{\mu}=(\varepsilon, \boldsymbol{k})$ and mass $m$, is detected, is given by the contraction between the lepton tensor and the hadron tensor, i.e.,

$$
\mathrm{d} \sigma=\frac{G^{2} \cos ^{2} \vartheta_{\mathrm{c}}}{2} 2 \pi L^{\mu \nu} W_{\mu \nu} \frac{\mathrm{d}^{3} k}{(2 \pi)^{3}},
$$

where $G \simeq 1.16639 \times 10^{-11} \mathrm{MeV}^{-2}$ is the Fermi constant and $\vartheta_{\mathrm{c}}$ is the Cabibbo angle $\left(\cos \vartheta_{\mathrm{c}} \simeq 0.9749\right)$.

The lepton tensor is

$$
L^{\mu \nu}=\frac{1}{2 \varepsilon_{i}} \frac{m}{\varepsilon} \sum_{\text {spin }} \bar{u}_{f} \gamma^{\mu}\left(1 \mp \gamma^{5}\right) u_{i} \bar{u}_{i}\left(1 \pm \gamma^{5}\right) \gamma^{\nu} u_{f},
$$

where the upper (lower) sign corresponds to neutrino (antineutrino) scattering. After projecting into the initial neutrino (antineutrino) and the final lepton state, one has

$$
L^{\mu \nu}=\frac{1}{2 \varepsilon_{i} \varepsilon} \operatorname{Tr}\left[\gamma \cdot k \gamma^{\mu}\left(1 \mp \gamma^{5}\right) \gamma \cdot k_{i} \gamma^{\nu}\right],
$$

which can be written, by separating the symmetrical and antisymmetrical components, as

$$
L^{\mu \nu}=\frac{2}{\varepsilon_{i} \varepsilon}\left[l_{S}^{\mu \nu} \mp l_{A}^{\mu \nu}\right],
$$

with

$$
\begin{aligned}
& l_{S}^{\mu \nu}=k_{i}^{\mu} k^{\nu}+k_{i}^{\nu} k^{\mu}-g^{\mu \nu} k_{i} \cdot k \\
& l_{A}^{\mu \nu}=i \epsilon^{\mu \nu \alpha \beta} k_{i \alpha} k_{\beta},
\end{aligned}
$$

where $\epsilon^{\mu \nu \alpha \beta}$ is the antisymmetric tensor with $\epsilon_{0123}=$ $-\epsilon^{0123}=1$.

Assuming the reference frame where the $z$-axis is parallel to the momentum transfer $\boldsymbol{q}=\boldsymbol{k}_{i}-\boldsymbol{k}$ and the $y$ axis is parallel to $\boldsymbol{k}_{i} \times \boldsymbol{k}$, the symmetrical components $l_{S}^{0 y}, l_{S}^{x y}, l_{S}^{z y}$, and the antisymmetrical ones $l_{A}^{0 x}, l_{A}^{x z}, l_{A}^{0 z}$, as well as those obtained from them by exchanging their indices, vanish.

The hadron tensor is given by bilinear products of the transition matrix elements of the nuclear weak chargedcurrent operator $J^{\mu}$ between the initial state $\left|\Psi_{0}\right\rangle$ of the nucleus, of energy $E_{0}$, and the final states $\left|\Psi_{\mathrm{f}}\right\rangle$, of energy $E_{\mathrm{f}}$, both eigenstates of the $(A+1)$-body Hamiltonian $H$, as

$$
\begin{array}{r}
W^{\mu \nu}(\omega, q)=\sum_{\mathrm{f}}\left\langle\Psi_{\mathrm{f}}\left|J^{\mu}(\boldsymbol{q})\right| \Psi_{0}\right\rangle \\
\times\left\langle\Psi_{0}\left|J^{\nu \dagger}(\boldsymbol{q})\right| \Psi_{\mathrm{f}}\right\rangle \delta\left(E_{0}+\omega-E_{\mathrm{f}}\right) .
\end{array}
$$

and involves an average over initial states and a sum over the undetected final states. The sum runs over the scattering states corresponding to all of the allowed asymptotic configurations and includes possible discrete states.

The transition matrix elements are calculated in the first order perturbation theory and in the impulse approximation, i.e., the incident neutrino interacts with only one nucleon while the other ones behave as spectators. The current operator is assumed to be adequately described as the sum of single-nucleon currents, corresponding to the weak charged current

$$
\begin{aligned}
j^{\mu} & =\left[F_{1}^{\mathrm{V}}\left(Q^{2}\right) \gamma^{\mu}+i \frac{\kappa}{2 M} F_{2}^{\mathrm{V}}\left(Q^{2}\right) \sigma^{\mu \nu} q_{\nu}\right. \\
& \left.-G_{\mathrm{A}}\left(Q^{2}\right) \gamma^{\mu} \gamma^{5}+F_{\mathrm{P}}\left(Q^{2}\right) q^{\mu} \gamma^{5}\right] \tau^{ \pm}
\end{aligned}
$$

where $\tau^{ \pm}$are the isospin operators, $\kappa$ is the anomalous part of the magnetic moment, $q^{\mu}=(\omega, \boldsymbol{q})$, with $Q^{2}=|\boldsymbol{q}|^{2}-\omega^{2}$, is the four-momentum transfer, and $\sigma^{\mu \nu}=(i / 2)\left[\gamma^{\mu}, \gamma^{\nu}\right] . F_{1}^{\mathrm{V}}$ and $F_{2}^{\mathrm{V}}$ are the isovector Dirac and Pauli nucleon form factors, which are taken from Ref. 21]. $G_{\mathrm{A}}$ and $F_{\mathrm{P}}$ are the axial and induced pseudoscalar form factors, which are usually parametrized as

$$
\begin{aligned}
G_{\mathrm{A}} & =\frac{g_{\mathrm{A}}}{\left(1+Q^{2} / M_{\mathrm{A}}^{2}\right)^{2}}, \\
F_{\mathrm{P}} & =\frac{2 M G_{\mathrm{A}}}{m_{\pi}^{2}+Q^{2}},
\end{aligned}
$$

where $g_{\mathrm{A}}=1.267, m_{\pi}$ is the pion mass, and $M_{\mathrm{A}} \simeq 1.032$ $\mathrm{GeV}$ is the axial mass.

The most general covariant form of the hadron tensor is obtained in terms of the two independent four-vectors of the problem, i.e. the four-momentum transfer $q^{\mu}$ and the four-momentum $P^{\mu}$ of the target. The symmetrical and antisymmetrical components of the tensor can therefore be written as

$$
\begin{aligned}
W_{S}^{\mu \nu} & =W_{1} g^{\mu \nu}+W_{2} q^{\mu} q^{\nu}+W_{3} P^{\mu} P^{\nu} \\
& +W_{4}\left(P^{\mu} q^{\nu}+q^{\mu} P^{\nu}\right) \\
W_{A}^{\mu \nu} & =W_{5}\left(P^{\mu} q^{\nu}-q^{\mu} P^{\nu}\right)+W_{6} \epsilon^{\mu \nu \alpha \beta} q_{\alpha} P_{\beta},
\end{aligned}
$$

where the invariant form factors $W_{i}$ are functions of the two scalars which can be formed with $q^{\mu}$ and $P^{\mu}$, i.e., $Q^{2}$ and $P \cdot q$. From Eq. (10), it is clear that in our reference frame $W^{0 x}, W^{0 y}, W^{x z}, W^{y z}$, and $W_{S}^{x y}$ vanish together with the tensor components obtained from them by exchanging their indices.

The inclusive cross section for the QE $\nu(\bar{\nu})$-nucleus scattering, obtained from the contraction between the lepton and hadron tensors, can therefore be written as 22

$$
\begin{aligned}
\frac{\mathrm{d} \sigma}{\mathrm{d} \varepsilon \mathrm{d} \Omega} & =k \varepsilon \frac{G^{2}}{4 \pi^{2}} \cos ^{2} \vartheta_{\mathrm{c}}\left[v_{0} R_{00}+v_{z z} R_{z z}\right. \\
& \left.-v_{0 z} R_{0 z}+v_{T} R_{T} \pm v_{x y} R_{x y}\right] .
\end{aligned}
$$


The coefficients $v$, obtained from the lepton tensor components, are

$$
\begin{aligned}
v_{0} & =1+\tilde{k} \cos \vartheta \\
v_{z z} & =1+\tilde{k} \cos \vartheta-2 \frac{\varepsilon_{i}|\boldsymbol{k}| \tilde{k}}{|\boldsymbol{q}|^{2}} \sin ^{2} \vartheta, \\
v_{0 z} & =\frac{\omega}{|\boldsymbol{q}|}(1+\tilde{k} \cos \vartheta)+\frac{m^{2}}{|\boldsymbol{q}| \varepsilon}, \\
v_{T} & =1-\tilde{k} \cos \vartheta+\frac{\varepsilon_{i}|\boldsymbol{k}| \tilde{k}}{|\boldsymbol{q}|^{2}} \sin ^{2} \vartheta \\
v_{x y} & =\frac{\varepsilon_{i}+\varepsilon}{|\boldsymbol{q}|}(1-\tilde{k} \cos \vartheta)-\frac{m^{2}}{|\boldsymbol{q}| \varepsilon},
\end{aligned}
$$

where $\tilde{k}=|\boldsymbol{k}| / \varepsilon, \vartheta$ is the lepton scattering angle, and $m$ the mass of the emitted lepton, e.g., the muon mass $\simeq 105.9 \mathrm{MeV}$. It was taken

$$
v_{T}=\frac{1}{2 \varepsilon_{i} \varepsilon}\left(l_{S}^{x x}+l_{S}^{y y}\right),
$$

taking advantage of the fact that $W^{x x}=W^{y y}$, as can be deduced from Eq. (10).

The response functions are given in terms of the components of the hadron tensor as

$$
\begin{aligned}
R_{00} & =W^{00} \\
R_{z z} & =W^{z z} \\
R_{0 z} & =W^{0 z}+W^{z 0}, \\
R_{T} & =W^{x x}+W^{y y} \\
R_{x y} & =i\left(W^{y x}-W^{x y}\right) .
\end{aligned}
$$

\section{THE RELATIVISTIC GREEN'S FUNCTION APPROACH}

All nuclear structure information is contained in the response functions and therefore in the hadron tensor. We apply here to the inclusive $\mathrm{QE} \nu(\bar{\nu})$-nucleus scattering the same relativistic approach [16] which was rather successful in reproducing the cross sections of the inclusive QE electron scattering. Here we recall only the main features of the model. More details can be found in Refs. 14, 15, 16]

For the inclusive process the components of the hadron tensor in Eq. (6) can equivalently be expressed as

$$
W^{\mu \nu}(\omega, q)=-\frac{1}{\pi} \operatorname{Im}\left\langle\Psi_{0}\left|J^{\nu \dagger}(\boldsymbol{q}) G\left(E_{\mathrm{f}}\right) J^{\mu}(\boldsymbol{q})\right| \Psi_{0}\right\rangle,
$$

in terms of the Green's function $G\left(E_{\mathrm{f}}\right)$ related to the nuclear Hamiltonian $H$, i.e.,

$$
G\left(E_{\mathrm{f}}\right)=\frac{1}{E_{\mathrm{f}}-H+i \eta} .
$$

Here and in all the equations involving $G$ the limit for $\eta \rightarrow+0$ is understood. It must be performed after calculating the matrix elements between normalizable states.
The current operator is assumed to be adequately described as the sum of the single-nucleon currents of Eq. (7).

It was shown in Refs. 14, 15, 16, 17] for the inclusive $\left(e, e^{\prime}\right)$ scattering that the components of the nuclear response in Eq. (15) can be written in terms of the singleparticle Green's function $\mathcal{G}(E)$, whose self-energy is the Feshbach's optical potential. An explicit calculation of the Green's function can be avoided by its spectral representation, which is based on a biorthogonal expansion in terms of the eigenfunctions of the non-Hermitian optical potential $\mathcal{V}$, and of its Hermitian conjugate $\mathcal{V}^{\dagger}$, i.e.,

$$
\begin{aligned}
{\left[\mathcal{E}-T-\mathcal{V}^{\dagger}(E)\right]\left|\chi_{\mathcal{E}}^{(-)}(E)\right\rangle } & =0, \\
{[\mathcal{E}-T-\mathcal{V}(E)]\left|\tilde{\chi}_{\mathcal{E}}^{(-)}(E)\right\rangle } & =0 .
\end{aligned}
$$

Note that $E$ and $\mathcal{E}$ are not necessarily the same. The spectral representation is

$$
\begin{aligned}
\mathcal{G}(E) & =\int_{M}^{\infty} \mathrm{d} \mathcal{E}\left|\tilde{\chi}_{\mathcal{E}}^{(-)}(E)\right\rangle \\
& \times \frac{1}{E-\mathcal{E}+i \eta}\left\langle\chi_{\mathcal{E}}^{(-)}(E)\right| .
\end{aligned}
$$

The hadron tensor can be reduced to a single-particle expression and can be written in an expanded form as

$$
\begin{aligned}
W^{\mu \nu}(\omega, q) & =-\frac{1}{\pi} \sum_{n} \operatorname{Im}\left[\int_{M}^{\infty} \mathrm{d} \mathcal{E} \frac{1}{E_{\mathrm{f}}-\varepsilon_{n}-\mathcal{E}+i \eta}\right. \\
& \left.\times T_{n}^{\mu \nu}\left(\mathcal{E}, E_{\mathrm{f}}-\varepsilon_{n}\right)\right]
\end{aligned}
$$

where $n$ denotes the eigenstate of the residual Hamiltonian of $A$ interacting nucleons related to the discrete eigenvalue $\varepsilon_{n}$ and

$$
\begin{aligned}
T_{n}^{\mu \nu}(\mathcal{E}, E) & =\lambda_{n}\left\langle\varphi_{n}\left|j^{\nu^{\dagger}}(\boldsymbol{q}) \sqrt{1-\mathcal{V}^{\prime}(E)}\right| \tilde{\chi}_{\mathcal{E}}^{(-)}(E)\right\rangle \\
& \times\left\langle\chi_{\mathcal{E}}^{(-)}(E)\left|\sqrt{1-\mathcal{V}^{\prime}(E)} j^{\mu}(\boldsymbol{q})\right| \varphi_{n}\right\rangle .
\end{aligned}
$$

$\lambda_{n}$ is the spectral strength of the hole state $\left|\varphi_{n}\right\rangle$, which is the normalized overlap integral between $\left|\Psi_{0}\right\rangle$ and $|n\rangle$, while the factor $\sqrt{1-\mathcal{V}^{\prime}(E)}$ was shown in Ref. 16] to account for interference effects between different channels and to allow the replacement of the mean field $\mathcal{V}$ by the phenomenological optical potential $\mathcal{V}_{\mathrm{L}}$.

After calculating the limit for $\eta \rightarrow+0$, Eq. (19) reads

$$
\begin{array}{r}
W^{\mu \nu}(\omega, q)=\sum_{n}\left[\operatorname{Re} T_{n}^{\mu \nu}\left(E_{\mathrm{f}}-\varepsilon_{n}, E_{\mathrm{f}}-\varepsilon_{n}\right)\right. \\
\left.-\frac{1}{\pi} \mathcal{P} \int_{M}^{\infty} \mathrm{d} \mathcal{E} \frac{1}{E_{\mathrm{f}}-\varepsilon_{n}-\mathcal{E}} \operatorname{Im} T_{n}^{\mu \nu}\left(\mathcal{E}, E_{\mathrm{f}}-\varepsilon_{n}\right)\right],
\end{array}
$$

where $\mathcal{P}$ denotes the principal value of the integral. Eq. (21) separately involves the real and imaginary parts of $T_{n}^{\mu \nu}$. 
The second matrix element in Eq. (20), with the inclusion of $\sqrt{\lambda_{n}}$ and disregarding the square root correction due to interference effects, is the transition amplitude for the single-nucleon knockout from a nucleus in the state $\left|\Psi_{0}\right\rangle$ leaving the residual nucleus in the state $|n\rangle$. The attenuation of its strength, mathematically due to the imaginary part of $\mathcal{V}^{\dagger}$, is related to the flux lost towards the channels different from $n$. In the inclusive response this attenuation must be compensated by a corresponding gain, due to the flux lost, towards the channel $n$, by the other final states asymptotically originated by the channels different from $n$. In the description provided by the spectral representation of Eq. (18), the compensation is performed by the first matrix element in the right hand side of Eq. (20), where the imaginary part of $\mathcal{V}$ has the effect of increasing the strength. Similar considerations can be made, on the purely mathematical ground, for the integral of Eq. (21), where the amplitudes involved in $T_{n}^{\mu \nu}$ have no evident physical meaning when $\mathcal{E} \neq E_{\mathrm{f}}-\varepsilon_{n}$. We want to stress here that in the Green's function approach it is just the imaginary part of $\mathcal{V}$ which accounts for the redistribution of the strength among different channels.

The cross sections and the response functions of the inclusive QE neutrino (antineutrino)-nucleus scattering are calculated from the single-particle expression of the hadron tensor in Eq. (21). After the replacement of the mean field $\mathcal{V}(E)$ by the empirical optical model potential $\mathcal{V}_{\mathrm{L}}(E)$, the matrix elements of the nuclear current operator in Eq. (20), which represent the main ingredients of the calculation, are of the same kind as those giving the transition amplitudes of the electron induced nucleon knockout reaction in the relativistic distorted wave impulse approximation (RDWIA) 24]. Thus, the same treatment can be used which was successfully applied to describe exclusive $\left(e, e^{\prime} p\right)$ and $(\gamma, p)$ data [24, 25]. Here, of course, the nuclear electromagnetic current must be replaced by the nuclear weak charged-current operator of Eq. (7) .

The relativistic final wave function is written, as in Refs. [16, 24], in terms of its upper component following the direct Pauli reduction scheme, i.e.,

$$
\chi_{\mathcal{E}}^{(-)}(E)=\left(\frac{1^{\Psi_{\mathrm{f}+}}}{M+\mathcal{E}+S^{\dagger}(E)-V^{\dagger}(E)} \boldsymbol{\sigma} \cdot \boldsymbol{p} \Psi_{\mathrm{f}+}\right),
$$

where $S(E)$ and $V(E)$ are the scalar and vector energydependent components of the relativistic optical potential for a nucleon with energy $E$ [26]. The upper component, $\Psi_{\mathrm{f}+}$, is related to a two-component spinor, $\Phi_{\mathrm{f}}$, which solves a Schrödinger-like equation containing equivalent central and spin-orbit potentials, obtained from the relativistic scalar and vector potentials [27, 28], i.e.,

$$
\begin{aligned}
\Psi_{\mathrm{f}+} & =\sqrt{D_{\mathcal{E}}^{\dagger}(E)} \Phi_{\mathrm{f}} \\
D_{\mathcal{E}}(E) & =1+\frac{S(E)-V(E)}{M+\mathcal{E}}
\end{aligned}
$$

where $D_{\mathcal{E}}(E)$ is the Darwin factor.

As no relativistic optical potentials are available for the bound states, the wave functions $\varphi_{n}$ are taken as the Dirac-Hartree solutions of a relativistic Lagrangian containing scalar and vector potentials [29, 30].

\section{RESULTS}

The calculations have been performed with the same bound state wave functions and optical potentials as in Refs. 16, 24, 25], where the RDWIA was able to reproduce $\left(e, e^{\prime} p\right),(\gamma, p)$, and $\left(e, e^{\prime}\right)$ data.

The relativistic bound state wave functions have been obtained from Ref. 29], where relativistic HartreeBogoliubov equations are solved in the context of a relativistic mean field theory and reproduce single-particle properties of several spherical and deformed nuclei 30]. The scattering state is calculated by means of the energy-dependent and A-dependent EDAD1 complex phenomenological optical potential of Ref. [26], which is fitted to proton elastic scattering data on several nuclei in an energy range up to $1040 \mathrm{MeV}$.

The initial states $\left|\varphi_{n}\right\rangle$ are taken as single-particle onehole states in the target. A pure shell model is assumed for the nuclear structure, i.e., we take a unitary spectral strength for each single-particle state and the sum runs over all the occupied states.

The results presented in the following contain the contribution of only the first term in Eq. (21). The calculation of the second term, which requires integration over all the eigenfunctions of the continuum spectrum of the optical potential, is a complicate task. Its contribution has been estimated to be small in the kinematics explored; hence, it is neglected in the present calculations.

In order to show up the effect of the optical potential on the inclusive reaction, the results obtained in the present approach are compared with those given by different approximations.

In the simplest approach the optical potential is neglected, i.e., $\mathcal{V}=\mathcal{V}^{\dagger}=0$ in Eq. (17), and the plane wave approximation is assumed for the final state wave functions $\chi^{(-)}$and $\tilde{\chi}^{(-)}$. In this plane wave impulse approximation (PWIA) FSI between the outgoing nucleon and the residual nucleus are completely neglected.

In another approach the imaginary part of the optical potential is neglected and only the real part is included. This approximation, that was sometimes used in the past, conserves the flux, but it is inconsistent with the exclusive process, where a complex optical potential must be used. Moreover, the use of a real optical potential is unsatisfactory from a theoretical point of view, since the optical potential has to be complex owing to the presence of open channels.

The partial contribution given to the inclusive process by the sum of all the integrated exclusive reactions with one-nucleon emission is also shown in the following for a 

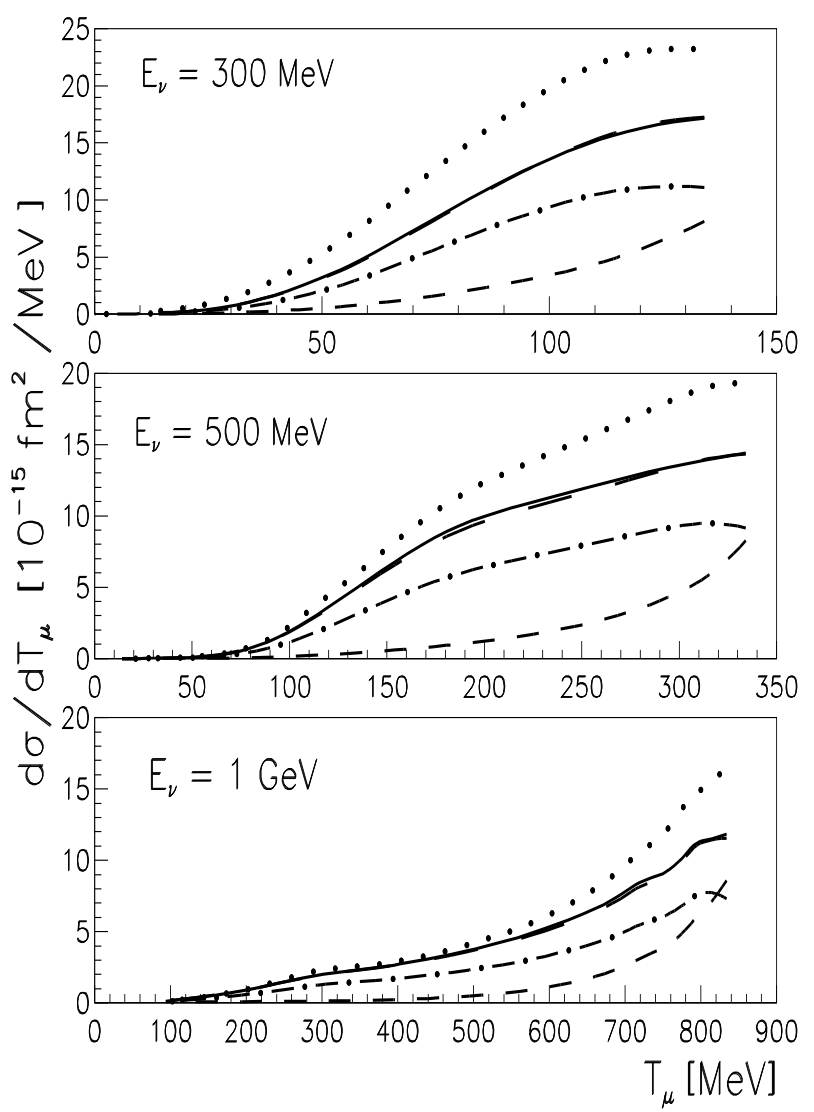

FIG. 1: The cross sections of the ${ }^{16} \mathrm{O}\left(\nu_{\mu}, \mu^{-}\right)$reaction, integrated over the muon angle, for $E_{\nu}=300$, 500, and 1000 $\mathrm{MeV}$. Solid lines represent the result of the Green's function approach, dotted lines give PWIA, long-dashed lines show the result with a real optical potential, and dot-dashed lines the contribution of the integrated exclusive reactions with onenucleon emission. Short dashed lines give the cross sections of the ${ }^{16} \mathrm{O}\left(\bar{\nu}_{\mu}, \mu^{+}\right)$reaction calculated with the Green's function approach.

comparison. In this case only the eigenfunctions $\chi^{(-)}$of $\mathcal{V}^{\dagger}$ are included and the imaginary part of the potential produces an absorption which does not conserve the total flux. We note that in the Green's function approach of Eqs. (20) and (21) FSI are treated, consistently with the exclusive process, by means of a complex optical potential. The imaginary part, however, does not produce a global loss of flux and it is responsible for the redistribution of the strength among different channels.

As a first study case we have considered the ${ }^{16} \mathrm{O}$ target nucleus, for which the adopted single-particle wave functions and optical potentials have given a good agreement between RDWIA calculations and $\left(e, e^{\prime} p\right),(\gamma, p)$, and $\left(e, e^{\prime}\right)$ data.

In Fig. [1 the cross sections of the ${ }^{16} \mathrm{O}\left(\nu_{\mu}, \mu^{-}\right)$reaction, integrated over the muon scattering angle, are displayed as a function of the muon kinetic energy $T_{\mu}$ for three different values of the incident neutrino energy $E_{\nu}=300$,
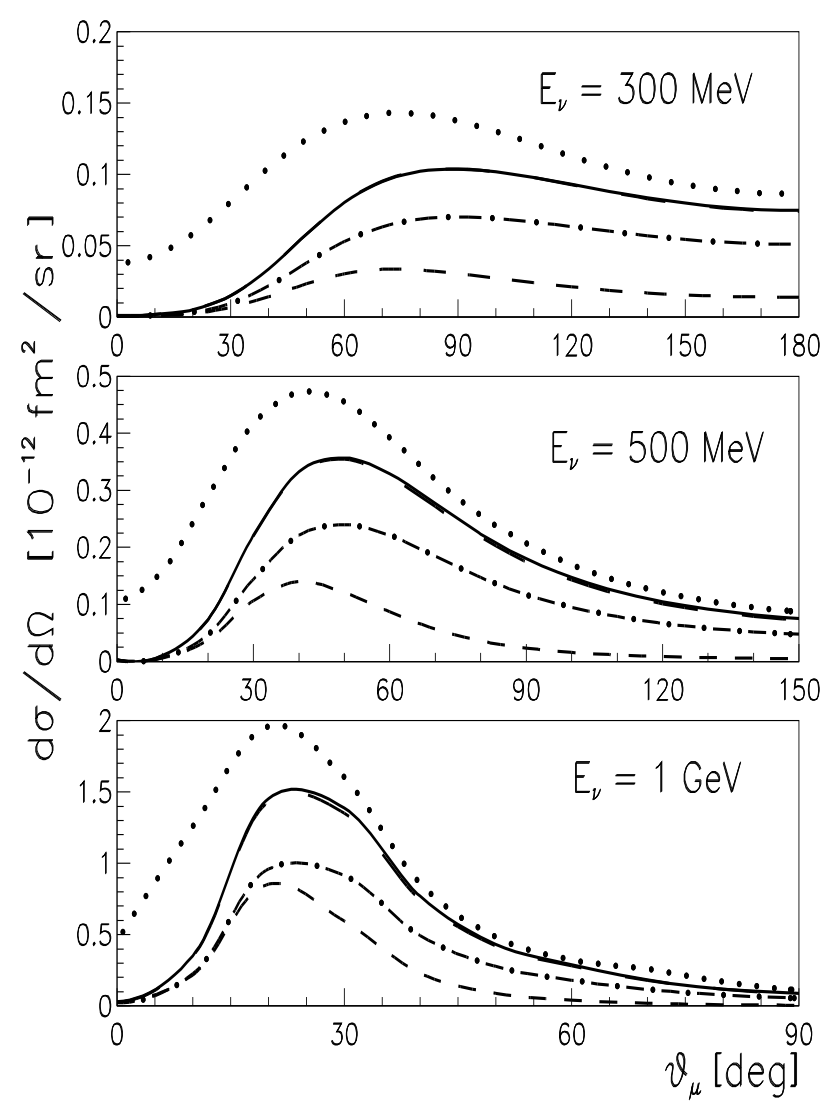

FIG. 2: The cross sections of the ${ }^{16} \mathrm{O}\left(\nu_{\mu}, \mu^{-}\right)$reaction, integrated over the muon energy, for $E_{\nu}=300,500$, and 1000 $\mathrm{MeV}$ as a function of the scattering angle of the outgoing muon $\theta_{\mu}$. Line convention as in Fig. 1]

500 and $1000 \mathrm{MeV}$. The behaviour of the calculated cross sections is similar for the different energies. The effect of the optical potential increases with $T_{\mu}$ and decreases increasing $E_{\nu}$. At $300 \mathrm{MeV}$, the result of the PWIA is much higher than the one of the Green's function approach, while at $1 \mathrm{GeV}$ the two results are almost the same but at the highest values of $T_{\mu}$. The sum of the exclusive one-nucleon emission cross sections is always much smaller than the complete result. The difference indicates the relevance of inelastic channels and is due to the loss of flux produced by the absorptive imaginary part of the optical potential. In contrast, the cross sections calculated with only the real part of the optical potential are practically the same as the ones obtained with the Green's function approach. Although the use of a complex optical potential is conceptually important from a theoretical point of view, the negligible differences given by the two results mean that the conservation of flux, that is fulfilled in both calculations, is the most important condition in the present situation. In contrast, significant differences are obtained with a real optical potential in the inclusive electron scattering [15, 16]. 


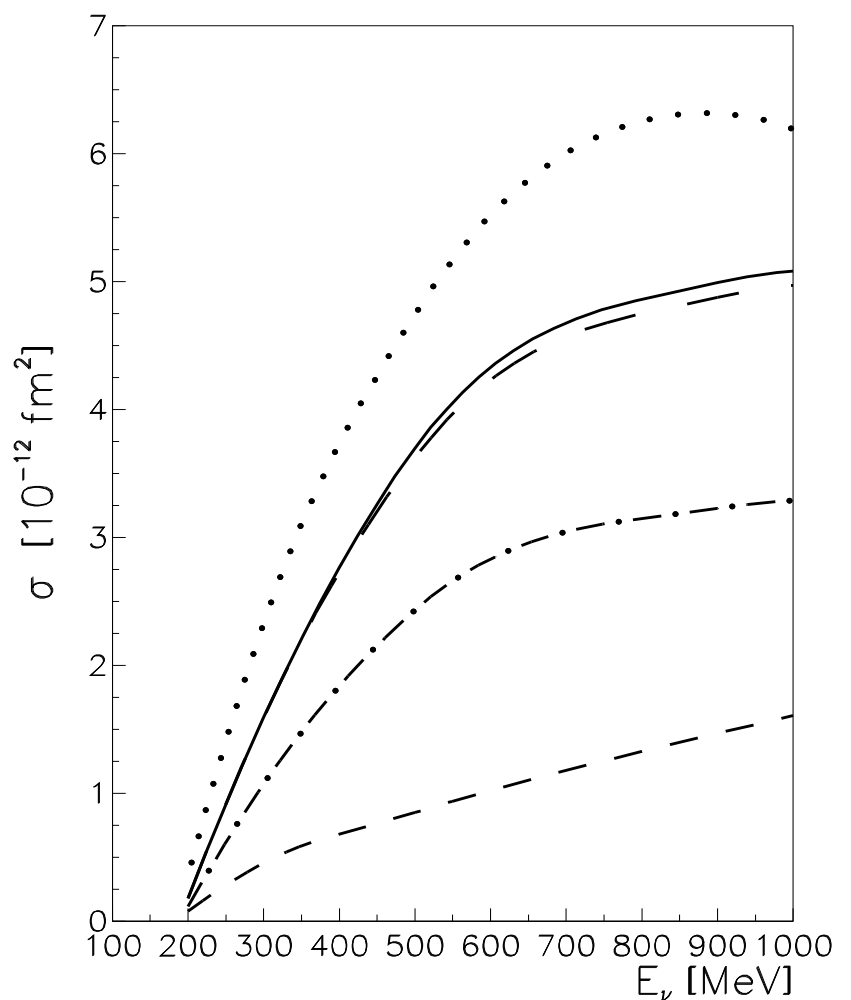

FIG. 3: The total cross section of the ${ }^{16} \mathrm{O}\left(\nu_{\mu}, \mu^{-}\right)$reaction, integrated over the muon energy and angle, in terms of the neutrino energy $E_{\nu}$. Line convention as in Fig. 1]

Qualitatively similar results are obtained in Fig. 2 where the cross sections integrated over the muon energy are displayed for $E_{\nu}=300,500$, and $1000 \mathrm{MeV}$ as a function of the scattering angle of the outgoing muon.

The global effect of FSI is clearly shown in Fig. 3 where the cross sections are integrated over the energy and the angle of the outgoing muon. The PWIA result is always much larger, while the loss of flux produced by the absorptive optical potential in the exclusive processes produces a too small cross section. In contrast, an optical potential with only a real component seems able to give a result comparable with the one of the Green's function approach. Small differences are found only at higher neutrino energies.

In Figs. 10 2 and 3 also the cross sections for the ${ }^{16} \mathrm{O}\left(\bar{\nu}_{\mu}, \mu^{+}\right)$reaction are shown for a comparison. They are always much smaller than the corresponding cross sections with an incident neutrino.

The different approaches have been compared with the experimental results of the LSND collaboration at Los Alamos for the ${ }^{12} \mathrm{C}\left(\nu_{\mu}, \mu^{-}\right)$reaction [1, 2, 3]. The calculations have been flux-averaged over the Los Alamos

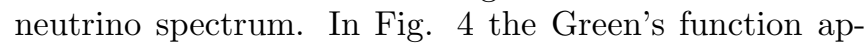
proach is normalized to the experimental data, while the other results are accordingly scaled. The shape of experimental data is reasonably reproduced. The flux-averaged

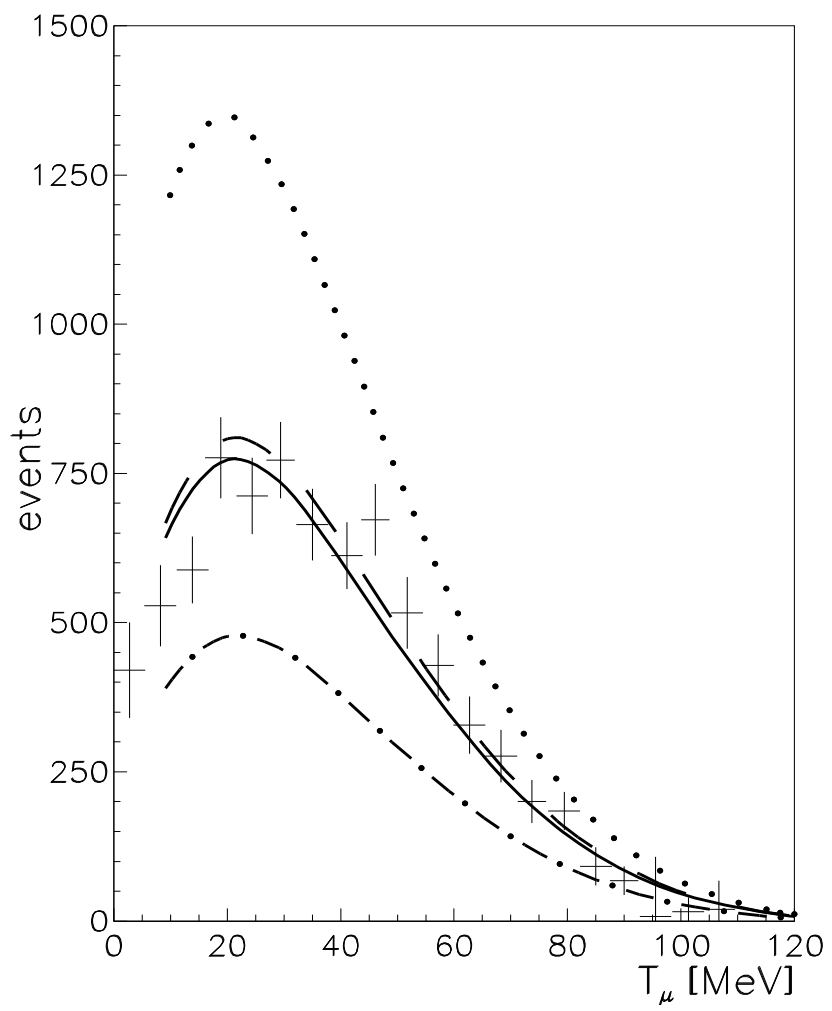

FIG. 4: The distribution of the muon kinetic energy for the inclusive ${ }^{12} \mathrm{C}\left(\nu_{\mu}, \mu^{-}\right)$reaction. Experimental data from Ref. [2]. The result of the Green's function approach is normalized to the experimental data. The other curves are scaled, accordingly. Line convention as in Fig. 1]

cross section integrated over the muon energy gives 11.15 $\times 10^{-40} \mathrm{~cm}^{2}$. At the low energy of the experiment, however, other processes, which are here not included, besides the quasielastic scattering can affect the inclusive reaction, in particular the excitation of the discrete states of ${ }^{12} \mathrm{C}$. The experimental value $(10.6 \pm 0.3 \pm 1.8) \times 10^{-40}$ $\mathrm{cm}^{2}[3$ is slightly overestimated. The results obtained by other calculations [4, 6, 7, 8, 10, 11, 13] give larger values.

\section{SUMMARY AND CONCLUSIONS}

We have applied to $\left(\nu_{\mu}, \mu^{-}\right)$and $\left(\bar{\nu}_{\mu}, \mu^{+}\right)$reactions an approach based on the spectralization of the singleparticle Green's function in terms of the eigenfunctions of the complex optical potential and of its Hermitian conjugate. This approach has proved to be rather successful in describing inclusive electron scattering. Its advantage stands in the fact that it is able to include in a simple way and keeping flux conservation the final state interactions by using an optical potential which is essential to reproduce exclusive electron knockout reactions.

The method is applied within a relativistic framework to weak charged-current reactions for an energy up to 1 
$\mathrm{GeV}$, where nuclear structure effects are important and dominant with respect to nucleon-resonance excitations. The reaction mechanism is assumed to be a direct one, where the incident neutrino interacts with only one neutron and a proton is emitted together with a negativecharged muon. A single-particle model is used to describe the structure of the nucleus and a sum over all single-particle occupied states is performed.

Calculations for the ${ }^{16} \mathrm{O}$ target nucleus have been presented at neutrino energies up to $1 \mathrm{GeV}$. The optical potential and flux conservation have a large effect on the cross sections. For ${ }^{12} \mathrm{C}$, the results averaged over the ex- perimental flux of neutrinos are compared with the available data. A fair agreement is obtained in arbitrary units. The integrated cross section results somewhat larger than the experimental value.

\section{Acknowledgments}

We would like to thank W.M. Alberico for useful discussions.
[1] M. Albert, et al., Phys. Rev. C 51, 1065 (1995).

[2] C. Athanassopoulos, et al., Phys. Rev. C 56, 2806 (1997).

[3] L.B. Auerbach, et al., Phys. Rev. C 66, 015501 (2002).

[4] S.L. Mintz and M. Pourkaviani, Nucl. Phys. A594, 346 (1995).

[5] H. Kim, J. Piekarewicz, and C.J. Horowitz, Phys. Rev. C 51, 2739 (1995).

[6] S.K. Singh, N.C. Mukhopadhyay, and E. Oset, Phys. Rev. C 57, 2687 (1998).

[7] A.C. Hayes and I.S. Towner, Phys. Rev. C 61, 044603 (2000).

[8] C. Volpe, N. Auerbach, G. Colò, T. Suzuki, and N. Van Giai, Phys. Rev. C 62, 015501 (2000).

[9] W.M. Alberico, M.B. Barbaro, S.M. Bilenky, J.A. Caballero, C. Giunti, C. Maieron, E. Moya de Guerra, and J.M. Udías, Nucl. Phys. A623, 471 (1997).

[10] E. Kolbe, K. Langanke, and P. Vogel, Nucl. Phys. A652, 91 (1999).

[11] N. Jachowicz, K. Heyde, J. Ryckebusch, and S. Rombouts, Phys. Rev. C 65, 025501 (2002).

[12] C. Bleve, G. Co', I. De Mitri, P. Bernardini, G. Mancarella, D. Martello, and A. Surdo, Astropart. Phys. 16, 145 (2001); G. Co', C. Bleve, I. De Mitri, and D. Martello, Nucl. Phys. Proc. Suppl. 112, 210 (2002).

[13] C. Maieron, M.C. Martínez, J.A. Caballero, and J.M. Udías, Phys. Rev. C 68, 048501 (2003).

[14] S. Boffi, C. Giusti, F. D. Pacati, and M. Radici, Electromagnetic Response of Atomic Nuclei, Oxford Studies in Nuclear Physics, Vol. 20 (Clarendon Press, Oxford, 1996); S. Boffi, C. Giusti, and F. D. Pacati, Phys. Rep. 226, 1 (1993).

[15] F. Capuzzi, C. Giusti, and F.D. Pacati, Nucl. Phys. A524, 681 (1991).
[16] A. Meucci, F. Capuzzi, C. Giusti, and F.D. Pacati, Phys. Rev. C 67, 054601 (2003).

[17] Y. Horikawa, F. Lenz, and N.C. Mukhopadhyay, Phys. Rev. C 22, 1680 (1980).

[18] C.R. Chinn, A. Picklesimer, and J.W. Van Orden, Phys. Rev. C 40, 790 (1989); 40, 1159 (1989).

[19] P.M. Boucher and J.W. Van Orden, Phys. Rev. C 43, 582 (1991).

[20] F. Capuzzi and C. Mahaux, Ann. Phys. (N.Y.) 254, 130 (1997).

[21] M.J. Musolf and T.W. Donnelly, Nucl. Phys. A546, 509 (1992).

[22] J.D. Walecka, in Muon Physics, Vol. II, edited by V.H. Hughes and C.S. Wu (Academic Press, New York, 1975), p. 113.

[23] S. Boffi and F. Capuzzi, Nucl. Phys. A351, 219 (1981).

[24] A. Meucci, C. Giusti, and F. D. Pacati, Phys. Rev. C 64, 014604 (2001).

[25] A. Meucci, C. Giusti, and F. D. Pacati, Phys. Rev. C 64, 064615 (2001).

[26] E.D. Cooper, S. Hama, B.C. Clark, and R.L. Mercer, Phys. Rev. C 47, 297 (1993).

[27] B.C. Clark, in Proceedings of the Workshop on Relativistic Dynamics and Quark-Nuclear Physics, edited by M.B. Johnson and A. Picklesimer (John Wiley \& Sons, New York, 1986), p. 302.

[28] M. Hedayati-Poor, J.I. Johansson, and H.S. Sherif, Nucl. Phys. A593, 377 (1995).

[29] W. Pöschl, D. Vretenar, and P. Ring, Comput. Phys. Commun. 103, 217 (1997).

[30] G.A. Lalazissis, J. König, and P. Ring, Phys. Rev. C 55, 540 (1997). 